\title{
Application of Non-Selective Herbicides in the Pre-Harvest of Wheat Damages Seed Quality
}

\author{
Glauber Monçon Fipke1, Thomas Newton Martin², Ubirajara Russi Nunes², \\ Evandro Ademir Deak ${ }^{1}$, Jessica Deolinda Leivas Stecca ${ }^{3}$, José Eduardo Minussi Winck ${ }^{4}$, \\ Luiz Fernando Teleken Grando ${ }^{5}$, Anderson da Costa Rossato ${ }^{5}$
}

\author{
${ }^{1}$ Post-Graduation Program in Agronomy at Department of Plant Science, Federal University of Santa Maria, Santa Maria, Brazil \\ ${ }^{2}$ Department of Plant Science, Federal University of Santa Maria, Santa Maria, Brazil \\ ${ }^{3}$ Laboratory of Seed Research and Teaching, Federal University of Santa Maria, Santa Maria, Brazil \\ ${ }^{4}$ Field Technical Manager, Sementes Lanes Ltda., São Gabriel, Brazil \\ ${ }^{5}$ Federal University of Santa Maria, Santa Maria, Brazil \\ Email:gm.fipke@hotmail.com,martin.ufsm@gmail.com,russinunes@yahoo.com.br, evandro.deak@hotmail.com, \\ jessica.stecca@yahoo.com.br, eduardo.winck@hotmail.com,luizf_grando@hotmail.com,ander.rs@hotmail.com
}

How to cite this paper: Fipke, G.M., Martin, T.N., Nunes, U.R., Deak, E.A., Stecca, J.D.L., Winck, J.E.M., Grando, L.F.T. and Rossato, A.C. (2018) Application of NonSelective Herbicides in the Pre-Harvest of Wheat Damages Seed Quality. American Journal of Plant Sciences, 9, 107-123. https://doi.org/10.4236/ajps.2018.91010

Received: December 1, 2017

Accepted: January 15, 2018

Published: January 19, 2018

Copyright $\odot 2018$ by authors and Scientific Research Publishing Inc. This work is licensed under the Creative Commons Attribution International License (CC BY 4.0).

http://creativecommons.org/licenses/by/4.0/

(c) (i) Open Access

\begin{abstract}
Pre-harvest desiccation in order to minimize the time of exposure to abiotic factors after physiological maturity is a practice used in the production of quality seeds. The aim of this work was to evaluate the physical, physiological and sanitary quality of wheat seeds from plants that received non-selective herbicide application at pre-harvest. For this, the treatments were applied on pre-harvest culture. A completely randomized design was applied. We use the herbicides (glufosinate-ammonium, glyphosate and paraquat), phenological stages of herbicide application (Z-83, Z-85, Z-87, Z-92) and additional treatment (without application). The routine tests that express the physical, physiological and sanitary integrity of seeds were evaluated. For herbicide treatments, there was an $8 \%$ and $26 \%$ reduction in germination under laboratory conditions for BRS Parrudo and Sinuelo, respectively. Soil emergence was $14 \%$ higher for the control treatment, not statistically different for the Z-87 and Z-92 stages and also for the herbicides glufosinate-ammonium and glyphosate for both cultivars. The application of non-selective herbicides on wheat pre-harvest impairs the physical and physiological quality and promotes faster deterioration. Vigor is reduced under stress conditions by cold test and accelerated aging. There is no influence on seed sanity.
\end{abstract}

\section{Keywords}

Glufosinate-Ammonium, Glyphosate, Paraquat, Triticum aestivum (L) 


\section{Introduction}

The use of wheat seeds [Triticum aestivum (L).] of high quality can provide a fast establishment of plants, thus, ensuring the number of plants per area which is the first component of the yield to be set. In the reproductive stage the occurrence of unfavorable environmental factors can affect the seed quality. Temperature and relative humidity, as well as, high levels of rainfall are commonplace in late spring in southern Brazil. Reducing time of exposure of seeds to stress caused by the environment is a viable strategy to ensure the physical, physiological and sanitary quality [1] [2]. Harvesting with seed moisture between 30\% $35 \%$ maintains the compounds of production, however, the germination of seeds is impaired [3]. Another alternative is to promote drying of the plant by applying non-selective herbicides in order to anticipate the harvest [4].

Herbicides can be used in the pre-harvest for weed control, as well as, promote drying of the plant and force the loss of the moisture content of the seed mass, promoting harvest more quickly and efficiently [5]. The non-selective herbicides are most often used before sowing or otherwise, in genetically modified cultivars with the restriction enzyme. The glufosinate-ammonium is of low mobility in the plant, acting in the course of glutamine synthetase, interfering with the metabolism of glutamic acid. Two to four hours after application the photosynthesis decreases and the plants are yellow and die within two to five days. This herbicide is recommended for use on pre-harvest of wheat, being the active ingredient in the composition of the single commercial product registered in Brazil for this purpose [6]. The glyphosate is a non-selective systemic herbicide that inhibits the 5-enolpyruvyl shikimate-3-phosphate synthase (EPSPS). Plant growth is inhibited soon after application, accompanied by leaf chlorosis and necrosis in general four to 10 days depending on the susceptibility of the deeds. This herbicide is recommended in several countries (United States, Canada, among others) for pre-harvest application, the most common being indicated the use when the seed moisture is close to $30 \%$. The paraquat is a contact herbicide that inhibits photosynthesis at photosystem I. The wilting and drying occurs rapidly within a few hours after application in full sunlight. Complete leaf necrosis occurs within one to three days. This herbicide can be used in pre-harvest of cultures, including soybeans. Must be applied three to four weeks before harvest, when $65 \%$ of the pods ripen and present seed moisture less than $30 \%$ [7] [8] [9].

The decision on the timing in pre-harvest for herbicide application is crucial to the success of the practice. Often, it is suitable when the plant is physiological maturity and has the lowest seed moisture as possible. In this way, the possibility of herbicide vascular tissue translocation is minimized, and the main route of contamination would be direct contact caused by spraying [10]. In the culture of wheat, [11] indicate the adoption of a practical criterion for the detection of physiological maturity. The node just below the spike may have green, unlike the rest of the plant. In field conditions, these characterizations can be very subjec- 
tive, which refers to the use of other types of plant characteristics. Researchers have used the color, consistency and degree of moisture of seeds, phenology, days after flowering, among others [5] [12] [13]. In order to harvest aid of seeds, the aim of this work to evaluate the physical, physiological and sanitary quality of wheat seeds from plants that have received application of non-selective herbicides in pre-harvest.

\section{Material and Methods}

\subsection{Obtaining Seeds}

Initially, the experiment was carried out in the field area at Department of Plant Science at the Federal University of Santa Maria (UFSM), Santa Maria, RS, Brazil $\left(29^{\circ} 42^{\prime} \mathrm{S}, 53^{\circ} 42^{\prime}\right.$ at an altitude of $116 \mathrm{~m}$ ). The sowing (300 viable seeds $\mathrm{m}^{2}$ ) ocurred on June 23, 2017, using the cultivars BRS Parrudo e TBIO Sinuelo. Mineral fertilization in sowing furrow (400 kg.ha ${ }^{-1}$ of $05-20-20 \mathrm{NPK}$ ) and topdressing ( $90 \mathrm{~kg} \cdot \mathrm{ha}^{-1}$ of urea) was calculated according to the interpretation of the soil analysis [14]. The pesticide management were carried out according to the technical information for the region [15]. During the pre-harvest, non-selective herbicides were applied (treatments), except for the additional treatment.

\subsection{Treatments Description}

The treatments were randomized in a factorial plus additional treatment $(3 \times 4+$ 1), totaling 13 treatments. On the first factor, were allocated the three herbicides, the second factor were allocated the four phenological stages of application of herbicides and the additional without application. As for the herbicides: glufosinate-ammonium [Finale ${ }^{\oplus}, 350 \mathrm{~g}$ active ingredient (a.i.) ha ${ }^{-1}$ ], glyphosate (Stinger $^{\oplus}, 1440$ g a.i. ha ${ }^{-1}$ ) e paraquat (Gramoxone $e^{\oplus}, 400 \mathrm{~g}^{\text {a.i. }} \mathrm{ha}^{-1}$ ). As the phenological stages of application of herbicides [16]: Z-83 (seed of early dough and green color, sticky content when crushing the caryopsis between fingers), Z-85 (seed of soft dough and light green color, when pressed with the finger nail impression not held), Z-87 (seed of hard dough and seed red clear color, when pressed with the finger nail impression held) and Z-92 (seed of too hard and red color, and may not be finger nail press). As for the additional treatment: there was no herbicide application, with de point of harvest determined by the physiological maturation of at least $95 \%$ of the spikes, pale color and decumbentcurvature. The herbicide treatments were applied using a $\mathrm{CO}_{2}$, pressurized backpack sprayer (200 kPa pressure) outfitted with four flat fan tips (Teejet XR 100.02), spaced at $0.5 \mathrm{~m}$, with a spray volume of $150 \mathrm{~L} \cdot \mathrm{ha}^{-1}$. Drinking water was used without the addition of adjuvants and surfactants.

\subsection{Drying, Storage and Characterization of Seeds}

This work was carried out in the Laboratory of Seed Research and Teaching (UFSM). The seeds were dried naturally by exposure to the sun until reaching the optimum moisture for storage $(13 \%-14 \%)$. The seeds were stored in paper 
packages in a cool and dry environment for a period of two months. The water content of seeds was determined using a laboratory oven at $105^{\circ} \mathrm{C} \pm 3^{\circ} \mathrm{C}$ for 24 hours. The 1000-seed mass was determined by measuring the mass of eight plots of 100 seeds [17] (Table 1 ).

\subsection{Physiological Evaluation}

Germination was evaluated by the first count of the germination test (FCGT) and germination (GER). We used 800 seeds, sown in germiteste moistened paper rolls to 2.5 times the mass of the dry paper and kept in the incubator at $20^{\circ} \mathrm{C}$. The evaluations were conducted at four and eight days after beginning of the test [17]. Normal seedlings were considered those who have a root system with at least $2 \mathrm{~mm}$ long and with coleoptile straight and well developed.

It was calculated the speed index (SI) the sowing of 800 -seeds in soil substrate. The daily count of the number of emerged seedlings until that number remained constant, being considered the emerged seedlings visible on the surface of the substrate. The emergencein soil (EMERs) was determined by counting the plants emergedin 20 days after sowing.

\subsection{Seedling Morphology}

Were measured the seedling shoot length (SL) and radicle (RL), selecting at random 20 normal seedlings after four days of germination test installation [18]. For the measurements was used a plastic ruler graduated. With those same selected plants was determined to seedling dry mass (SDM). The seedlings were deposited in paper packages, taken to a laboratory oven at $70^{\circ} \mathrm{C}$ until reaching a

Table 1. Description of the characteristics of the seed cultivars.

\begin{tabular}{|c|c|c|c|c|c|c|c|c|}
\hline \multirow[b]{2}{*}{$\mathrm{HER}^{*}$} & \multicolumn{4}{|c|}{-BRS Parrudo- } & \multicolumn{4}{|c|}{-TBIO Sinuelo- } \\
\hline & GLU & GLY & PAR & TWA & GLU & GLY & PAR & TWA \\
\hline PS & \multicolumn{8}{|c|}{ Watercontent (\%) } \\
\hline Z-83 & 14.3 & 14.6 & 14.2 & 13.6 & 13.9 & 13.9 & 14.0 & 14.3 \\
\hline Z-85 & 14.1 & 14.3 & 14.2 & & 13.9 & 14.6 & 14.4 & \\
\hline Z-87 & 13.3 & 13.8 & 14.1 & & 13.9 & 14.2 & 14.4 & \\
\hline \multirow[t]{2}{*}{ Z-92 } & 13.3 & 13.8 & 13.6 & & 14.0 & 14.0 & 14.4 & \\
\hline & \multicolumn{8}{|c|}{ 1000-seedmass (g) } \\
\hline Z-83 & 36.0 & 37.4 & 36.6 & 39.0 & 30.0 & 31.8 & 29.6 & 35.8 \\
\hline Z-85 & 37.3 & 38.1 & 37.0 & & 34.4 & 35.1 & 33.1 & \\
\hline Z-87 & 38.8 & 39.4 & 38.3 & & 35.0 & 35.0 & 35.6 & \\
\hline Z-92 & 39.8 & 38.2 & 38.4 & & 37.0 & 36.0 & 36.9 & \\
\hline
\end{tabular}

${ }^{\star}$ Herbicides (HER), phenological stage (PS), glufosinate-ammonium (GLU), glyphosate (GLY), paraquat (PAR), treatment without application (TWA). The dates October 24, October 28, November 01, November 06 and November 14, represents the application of herbicides on Z-83, Z-85, Z-87, Z-92 and harvesting of the TWA, respectively. 
constant weight [19].

These characters were evaluated with the plants emerged in soil, by selecting 20 -normal randomly plants with 20 days. Thus, it was determined the shoot lenght (SLs) and root (RLs) and the plant dry mass (PDMs) emerged in soil [20].

\subsection{Seed Vigor in Stress Conditions}

Germination by cold test (CT) was done according to methodology described for germination testing, adapting to the conditions of time and temperature in $5^{\circ} \mathrm{C}$ for seven days, respectively [18]. After this period, the incubator was regulated to $20^{\circ} \mathrm{C}$ for four more days, being evaluated the 11 th day. Germination by accelerated aging test (AA) was done initially in transparent plastic germination boxes (gerbox) containing $40 \mathrm{~mL}$ of distilled water at the bottom. An aluminum screen was then fixed at the upper edge of each gerbox where seed of each subsample were uniformly distributed on a single layer. The sets (gerbox + seeds) were subsequently placed into a controlled environmental chamber (at $43^{\circ} \mathrm{C}$ ) during $48 \mathrm{~h}$. After that period, the seeds of each gerbox were subjected to first count of the germination test as previously described.

\subsection{Physical Evaluation}

The indirect measurement of physical integrity was made by electrical conductivity (EC). Four replicates were used of 50-seeds with weight set, deposited in beakers and immersed in $75 \mathrm{~mL}$ of distilled and deionized water, taken the incubator at $20^{\circ} \mathrm{C}$, for a period of six hours. After this period, a conductivimeter was then used to measure the electrical conductivity of the solution [21].

\subsection{Sanitary Evaluation}

The incidence of pathogens was evaluated by blotter test (BT).The incidence of pathogens was evaluated by blotter spot test (BT). 200 seeds were used, divided into four repetitions and placed in gerbox, seed dispersal on two sheets of paper germiteste sterile and moistened with distilled and autoclaved water in proportion to 2.5 times your weight [22]. A solution of $1.05 \%$ sodium hypochlorite was used for an asepsis of the boxes. The boxes were kept in the incubator $\left(20^{\circ} \mathrm{C}\right)$ in 12 hours of lighting (with fluorescent lamps) and 12 hours of darkness, for seven days. After this period, fungi were evaluated in seeds, with the aid of binocular microscope loupe based on the morphology of propagative structures of pathogens [23].

\subsection{Data Analysis}

For the analysis, a completely randomized design was used and the tested cultivars were evaluated separately. The data were verified and met the mathematical model assumptions for errors normality [Shapiro-Wilk $(\mathrm{P} \leq 0.05)$ ] and homogeneity of the variances [Bartlett $(\mathrm{P} \leq 0.05)$ ]. A subsequent analysis of variance [F-test $(\mathrm{P} \leq 0.05)]$ was conducted, by comparing the degrees of freedom of the 
treatments versus the treatment without application [Scheffé $(\mathrm{P} \leq 0.05)]$ and the complementary grouping test [Scott-Knott $(\mathrm{P} \leq 0.05)$ ]. Software Action ${ }^{\oplus}$ (Estatcamp, São Carlos, SP, Brazil), Genes ${ }^{\circledR}$ [24], Sisvar ${ }^{\circledR}[25]$ were used.

\section{Results}

About the interactions, it was verified that most of them were significant, as well as, contrasts with results favorable to the treatment without application (TWA) (Table 2 and Table 3). The characters: first count of the germination test (FCGT), germination (GER), speed index (SI), emergence in soil (EMERs), cold test (CT), accelerated aging test (AA) and electrical conductivity (EC) did not meet the normality of errors by the Shapiro-Wilk test $(\mathrm{P} \leq 0.05)$; To perform the F-test, we used the angular transformation (Arco seno $\sqrt{x / 100}$ ). The blotter test (BT) did not meet the assumption of the normality of the errors by the Shapiro-Wilk test $(\mathrm{P} \leq 0.05)$; For the F-test, we used the square root transformation $(\sqrt{x+0.5})$. The tables are represented by the averages (Tables $4-7$ ).

Table 2. Summary of the analysis of variance represented by the mean squares and estimates of the contrasts of treatments with non-selective herbicides applied in the wheat pre-harvest (BRS Parrudo) versus the treatment without application of the characters related to the physical and physiological quality of seeds.

\begin{tabular}{|c|c|c|c|c|c|c|c|c|c|c|c|c|c|c|}
\hline S.V. ${ }^{1}$ & D.F. & FCGT & GER & SL & $\mathrm{RL}$ & SDM & SI & EMERs & SLs & RLs & PDMs & $\mathrm{CT}$ & $\mathrm{AA}$ & $\mathrm{EC}$ \\
\hline Treatments & 12 & $503.6^{* *}$ & $321.3^{* *}$ & $1.16^{* *}$ & $8.14^{* *}$ & $18.9^{* *}$ & $3.48^{\star *}$ & $343.4^{* *}$ & $4.24^{\star *}$ & $5.03^{* *}$ & $156.9^{* *}$ & $327.8^{* *}$ & $435.4^{* *}$ & $54.2^{\star * *}$ \\
\hline Fatorial & 11 & $517.1^{\star *}$ & $340.8^{\star *}$ & $1.16^{\star *}$ & $8.71^{\star *}$ & $19.8^{* *}$ & $3.64^{* *}$ & $359.2^{* *}$ & $4.36^{\star *}$ & $4.01^{\star *}$ & $165.3^{* *}$ & $352.5^{\star *}$ & $473.9^{\star *}$ & $54.6^{* *}$ \\
\hline Contrast & 1 & $354.8^{* *}$ & $106.3^{* *}$ & $1.08^{\mathrm{ns}}$ & $1.84^{* *}$ & $8.6^{\mathrm{ns}}$ & $1.68^{*}$ & $170.3^{*}$ & $2.96^{\mathrm{ns}}$ & $16.30^{* *}$ & $65.1^{\mathrm{ns}}$ & $55.9^{\mathrm{ns}}$ & $12.0^{\mathrm{ns}}$ & $49.0^{* *}$ \\
\hline Error & 39 & 7.8 & 5.7 & 0.07 & 0.17 & 4.6 & 0.26 & 31.8 & 0.94 & 1.28 & 26.1 & 22.1 & 9.1 & 0.8 \\
\hline A (Herb.) & 3 & 1076.6 & 658.1 & 1.74 & 17.33 & 9.2 & 3.41 & 900.8 & 1.87 & 3.28 & 135.9 & 407.3 & 702.4 & 142.2 \\
\hline D (Phen.St.) & 2 & 746.8 & 404.7 & 0.64 & 13.09 & 8.5 & 3.71 & 465.2 & 0.83 & 6.76 & 386.7 & 718.7 & 721.2 & 49.7 \\
\hline$A \times D$ & 6 & 160.9 & 160.8 & 1.06 & 2.96 & 28.8 & 3.75 & 53.1 & 6.79 & 3.46 & 106.2 & 203.1 & 277.2 & 12.5 \\
\hline Average & & 77.2 & 84.6 & 3.2 & 5.5 & 30.2 & 1.80 & 75.7 & 12.2 & 12.9 & 58.5 & 90.1 & 74.5 & 12.7 \\
\hline CV (\%) & & 4.5 & 3.5 & 8.2 & 7.6 & 7.2 & 6.64 & 9.2 & 8.0 & 8.8 & 8.7 & 6.4 & 5.0 & 4.3 \\
\hline & & \multicolumn{13}{|c|}{-Contrasts- } \\
\hline Treatments & & $9.80^{* *}$ & $5.36^{* *}$ & $0.45^{\mathrm{ns}}$ & $0.70^{* *}$ & $1.53^{\mathrm{ns}}$ & $0.67^{\star *}$ & $6.79^{\star *}$ & $0.89^{\text {ns }}$ & $2.10^{* *}$ & $4.20^{\mathrm{ns}}$ & $3.89^{\text {ns }}$ & $1.80^{\mathrm{ns}}$ & $-3.64^{\star *}$ \\
\hline glufosinate & & $3.96^{* *}$ & $1.37^{\mathrm{ns}}$ & $0.30^{*}$ & $-0.27^{\mathrm{ns}}$ & $2.34^{\mathrm{ns}}$ & $0.60^{\mathrm{ns}}$ & $4.65^{\mathrm{ns}}$ & $0.67^{\mathrm{ns}}$ & $2.75^{\star *}$ & $4.30^{\mathrm{ns}}$ & $-2.34^{\mathrm{ns}}$ & $-1.61^{\mathrm{ns}}$ & $-2.04^{\star *}$ \\
\hline glyphosate & & $8.12^{\star *}$ & $3.70^{\star *}$ & $0.68^{\star \star}$ & $0.89^{\star *}$ & $0.93^{\mathrm{ns}}$ & $0.23^{\text {ns }}$ & $2.7^{\mathrm{ns}}$ & $0.87^{\mathrm{ns}}$ & $2.10^{\star *}$ & $-0.76^{\mathrm{ns}}$ & $3.05^{\mathrm{ns}}$ & $-2.50^{\mathrm{ns}}$ & $-5.53^{\star \star}$ \\
\hline paraquat & & $17.31^{\star *}$ & $11.01^{\star *}$ & $0.38^{\star *}$ & $1.50^{\star *}$ & $1.32^{\mathrm{ns}}$ & $1.18^{\star *}$ & $12.92^{\star *}$ & $1.13^{\mathrm{ns}}$ & $1.45^{\mathrm{ns}}$ & $9.06^{* *}$ & $10.97^{\star *}$ & $9.54^{\star \star}$ & $-3.36^{\star *}$ \\
\hline Z-83 & & $22.38^{* *}$ & $15.46^{* *}$ & $0.97^{\star *}$ & $2.21^{* *}$ & $0.65^{\mathrm{ns}}$ & $1.45^{\star *}$ & $18.55^{\star *}$ & $1.18^{\mathrm{ns}}$ & $2.77^{\star \star}$ & $7.75^{\mathrm{ns}}$ & $11.36^{\star *}$ & $13.11^{\star *}$ & $-6.34^{\star \star}$ \\
\hline Z-85 & & $10.95^{\star *}$ & $4.07^{\star \star}$ & $0.37^{\star *}$ & $1.14^{\star *}$ & $2.02^{\mathrm{ns}}$ & $0.36^{\mathrm{ns}}$ & $5.82^{\mathrm{ns}}$ & $0.59^{\text {ns }}$ & $2.23^{* *}$ & $0.96^{\mathrm{ns}}$ & $2.14^{\mathrm{ns}}$ & $-1.35^{\mathrm{ns}}$ & $-6.89^{\star *}$ \\
\hline Z-87 & & $5.70^{* *}$ & $4.29^{\star \star}$ & $0.42^{* *}$ & $-0.26^{\text {ns }}$ & $2.50^{\mathrm{ns}}$ & $0.31^{\mathrm{ns}}$ & $5.10^{\mathrm{ns}}$ & $1.28^{\mathrm{ns}}$ & $1.58^{\mathrm{ns}}$ & $1.71^{\mathrm{ns}}$ & $4.68^{\mathrm{ns}}$ & $-0.72^{\mathrm{ns}}$ & $-0.52^{\mathrm{ns}}$ \\
\hline Z-92 & & $0.17^{\mathrm{ns}}$ & $-2.36^{\mathrm{ns}}$ & $0.05^{\mathrm{ns}}$ & $-0.26^{\mathrm{ns}}$ & $0.95^{\mathrm{ns}}$ & $0.56^{\mathrm{ns}}$ & $-2.32^{\mathrm{ns}}$ & $0.52^{\mathrm{ns}}$ & $1.81^{\mathrm{ns}}$ & $6.36^{\mathrm{ns}}$ & $-2.61^{\mathrm{ns}}$ & $-3.79^{\mathrm{ns}}$ & $-0.81^{\mathrm{ns}}$ \\
\hline
\end{tabular}

${ }^{1}$ Source of variation (S.V.), degree of freedom (D.F.), coefficient of variation (CV\%). Characters: first count of germination test (FCGT) and germination (GER), seedling shoot length (SL) and radicle (RL), seedling dry mass (SDM), speed index (SI), emergence in soil (EMERs), plant shoot length (SLs) and root (RLs) emerged in soil, dry mass of plants emerged in soil (PDMs), cold test (CT), accelerated aging test (AA), electrical conductivity (EC); not significant, $5 \%$ and $1 \%$ statistical difference by F-test $\left({ }^{\mathrm{ns}}\right.$, ${ }^{*}$ and ${ }^{* *}$, respectively). Estimates of the contrasts [Scheffé $\left.(\mathrm{P} \leq 0.05)\right]$ with positive numbers are favorable to treatment without application or negative to the treatments with herbicides. 
Table 3. Summary of the analysis of variance represented by the mean squares and estimates of the contrasts of treatments with non-selective herbicides applied in the wheat pre-harvest (TBIO Sinuelo) versus the treatment without application of the characters related to the physical and physiological quality of seeds.

\begin{tabular}{|c|c|c|c|c|c|c|c|c|c|c|c|c|c|c|}
\hline S.V. ${ }^{1}$ & D.F. & FCGT & GER & SL & $\mathrm{RL}$ & SDM & SI & EMERs & SLs & RLs & PDMs & $\mathrm{CT}$ & $\mathrm{AA}$ & $\mathrm{EC}$ \\
\hline Treatments & 12 & $1105.7^{\star \star}$ & $791.0^{\star \star}$ & $0.95^{\star *}$ & $9.34^{* *}$ & $14.8^{* *}$ & $8.63^{\star \star}$ & $537.1^{\star *}$ & $6.65^{\star *}$ & $15.91^{\star *}$ & $130.1^{\star *}$ & $890.8^{\star \star}$ & $687.3^{* *}$ & $66.5^{\star *}$ \\
\hline Fatorial & 11 & $1078.6^{\star *}$ & $800.7^{* *}$ & $0.88^{\star *}$ & $9.45^{* *}$ & $15.9^{* *}$ & $9.07^{\star *}$ & $576.3^{* *}$ & $7.24^{* *}$ & $16.66^{* *}$ & $141.8^{* *}$ & $875.0^{* *}$ & $680.7^{* *}$ & $68.2^{* *}$ \\
\hline Contrast & 1 & $1403.7^{\star *}$ & $684.1^{\star *}$ & $1.75^{\star \star}$ & $8.15^{\star \star}$ & $2.9^{\mathrm{ns}}$ & $3.79^{\star *}$ & $105.9^{\star *}$ & $0.15^{\mathrm{ns}}$ & $7.66^{\mathrm{ns}}$ & $1.4^{\mathrm{ns}}$ & $1063.8^{\star *}$ & $760.0^{* *}$ & $48.8^{\star *}$ \\
\hline Error & 39 & 5.9 & 8.7 & 0.10 & 0.20 & 3.6 & 0.23 & 4.5 & 2.00 & 2.27 & 22.8 & 27.4 & 14.5 & 0.9 \\
\hline A (Herb.) & 3 & 2624.1 & 1879.9 & 2.15 & 12.73 & 46.7 & 14.03 & 1105.4 & 23.87 & 22.72 & 468.7 & 2002.1 & 1027.2 & 213.5 \\
\hline D (Phen.St.) & 2 & 1311.9 & 1098.4 & 0.85 & 28.17 & 4.4 & 15.86 & 843.6 & 0.26 & 39.74 & 23.0 & 1292.8 & 1459.3 & 36.9 \\
\hline$A \times D$ & 6 & 228.1 & 162.0 & 0.26 & 1.57 & 4.4 & 4.34 & 222.6 & 1.26 & 5.95 & 17.9 & 172.2 & 247.9 & 5.9 \\
\hline Average & & 66.6 & 74.4 & 2.9 & 4.4 & 27.2 & 1.37 & 62.3 & 11.1 & 11.0 & 48.5 & 78.1 & 51.2 & 12.1 \\
\hline CV (\%) & & 4.4 & 4.9 & 11.1 & 10.3 & 7.0 & 7.34 & 4.1 & 12.7 & 13.5 & 9.8 & 8.2 & 8.6 & 4.8 \\
\hline \multicolumn{15}{|c|}{-Contrasts- } \\
\hline Treatments & & $19.49^{\star *}$ & $13.61^{\star \star}$ & $0.68^{\star *}$ & $1.48^{\star *}$ & $-0.89^{\text {ns }}$ & $1.01^{\star *}$ & $5.35^{\star *}$ & $0.20^{\mathrm{ns}}$ & $-1.44^{\mathrm{ns}}$ & $0.61^{\mathrm{ns}}$ & $16.97^{\star *}$ & $14.34^{\star *}$ & $-3.63^{* *}$ \\
\hline glufosinate & & $11.27^{\star \star}$ & $5.16^{\star *}$ & $0.51^{\star *}$ & $-0.04^{\mathrm{ns}}$ & $-1.31^{\mathrm{ns}}$ & $0.44^{\mathrm{ns}}$ & $0.74^{\mathrm{ns}}$ & $0.10^{\mathrm{ns}}$ & $-3.26^{* *}$ & $0.76^{\mathrm{ns}}$ & $7.56^{\star *}$ & $7.89^{* *}$ & $-2.20^{\star *}$ \\
\hline glyphosate & & $18.00^{\star *}$ & $13.94^{\star \star}$ & $0.94^{\star *}$ & $2.22^{\star *}$ & $-0.30^{\mathrm{ns}}$ & $0.43^{\text {ns }}$ & $1.59^{\text {ns }}$ & $0.16^{\mathrm{ns}}$ & $-0.54^{\mathrm{ns}}$ & $1.73^{\mathrm{ns}}$ & $17.88^{\star *}$ & $9.83^{\star *}$ & $-5.22^{\star *}$ \\
\hline paraquat & & $29.20^{\star *}$ & $21.72^{\star \star}$ & $0.60^{\star *}$ & $2.28^{\star *}$ & $-1.05^{\mathrm{ns}}$ & $2.16^{\star *}$ & $13.72^{\star \star}$ & $0.34^{\mathrm{ns}}$ & $-0.51^{\mathrm{ns}}$ & $-0.65^{\mathrm{ns}}$ & $25.47^{\star *}$ & $25.31^{\star \star}$ & $-3.47^{\star *}$ \\
\hline $\mathrm{Z}-83$ & & $37.05^{\star *}$ & $29.45^{\star *}$ & $1.26^{* *}$ & $2.48^{* *}$ & $-0.34^{\mathrm{ns}}$ & $2.28^{\star *}$ & $16.27^{* *}$ & $1.82^{\mathrm{ns}}$ & $-0.08^{\mathrm{ns}}$ & $7.10^{\mathrm{ns}}$ & $32.49^{* *}$ & $25.92^{\star *}$ & $-6.75^{\star *}$ \\
\hline Z-85 & & $25.64^{\star \star}$ & $17.33^{* *}$ & $0.74^{* *}$ & $2.26^{* *}$ & $0.10^{\mathrm{ns}}$ & $1.49^{* *}$ & $9.50^{* *}$ & $0.90^{\mathrm{ns}}$ & $-0.43^{\mathrm{ns}}$ & $4.57^{\mathrm{ns}}$ & $22.47^{\star *}$ & $16.33^{* *}$ & $-7.77^{\star *}$ \\
\hline $\mathrm{Z}-87$ & & $11.47^{\star \star}$ & $6.45^{\star *}$ & $0.32^{\text {ns }}$ & $0.65^{\mathrm{ns}}$ & $0.48^{\mathrm{ns}}$ & $0.40^{\mathrm{ns}}$ & $1.47^{\mathrm{ns}}$ & $-1.25^{\mathrm{ns}}$ & $-2.74^{\star \star}$ & $-6.19^{\mathrm{ns}}$ & $8.54^{\star *}$ & $11.23^{\star *}$ & $0.33^{\mathrm{ns}}$ \\
\hline Z-92 & & $3.82^{\mathrm{ns}}$ & $1.20^{\mathrm{ns}}$ & $0.42^{\mathrm{ns}}$ & $0.54^{\mathrm{ns}}$ & $-3.80^{\mathrm{ns}}$ & $-0.12^{\mathrm{ns}}$ & $-5.82^{\mathrm{ns}}$ & $-0.65^{\mathrm{ns}}$ & $-2.49^{* *}$ & $-3.00^{\mathrm{ns}}$ & $4.38^{\mathrm{ns}}$ & $3.89^{\mathrm{ns}}$ & $-0.35^{\mathrm{ns}}$ \\
\hline
\end{tabular}

${ }^{1}$ Source of variation (S.V.), degree of freedom (D.F.), coefficient of variation (CV\%). Characters: first count of germination test (FCGT) and germination (GER), seedling shoot length (SL) and radicle (RL), seedling dry mass (SDM), speed index (SI), emergence in soil (EMERs), plant shoot length (SLs) and root (RLs) emerged in soil, dry mass of plants emerged in soil (PDMs), cold test (CT), accelerated aging test (AA), electrical conductivity (EC); not significant, $5 \%$ and $1 \%$ statistical difference by F-test $\left({ }^{\text {ns }},{ }^{*}\right.$ and ${ }^{* *}$, respectively). Estimates of the contrasts [Scheffé $\left.(\mathrm{P} \leq 0.05)\right]$ with positive numbers are favorable to treatment without application or negative to the treatments with herbicides.

\subsection{BRS Parrudo}

The first count of the germination test (FCGT) was $18 \%$ higher than for the treatment without application (TWA), differed statistically of the treatments with herbicides, except for the Z-92 stage (Table 4) The most efficient application stage was Z-92 for all the herbicides tested and reduction of this character with the anticipation of application to earlier stages. The glufosinate-ammonium presented $8 \%$ and $31 \%$ increase in relation to glyphosate and paraquat, respectively. For herbicide treatments, there were an $8 \%$ decrease in germination test (GER), differing statistically from the TWA. There was reduction for the first three application stages. In the Z-83 stage, glyphosate (73.3\%) and paraquat (46.0\%) were negatively characterized because germination averages below the minimum limit allowed by legislation (80.0\%).

The seedling shoot length (SL, $3.2 \mathrm{~cm}$ ) and dry mass (SDM, $30.1 \mathrm{mg}$ ) of herbicide treatments did not differ statistically from the TWA $(3.7 \mathrm{~cm}$ and $31.6 \mathrm{mg}$, 
Table 4. Characters related to seedling morphology and physiological quality of wheat (BRS Parrudo and TBIO Sinuelo) with application of non-selective herbicide in the pre-harvest.

\begin{tabular}{|c|c|c|c|c|c|c|c|c|c|c|c|c|c|c|}
\hline \multirow[b]{2}{*}{ HER $^{1}$} & \multicolumn{7}{|c|}{-BRS Parrudo- } & \multicolumn{7}{|c|}{-TBIO Sinuelo- } \\
\hline & \multicolumn{2}{|l|}{ GLU } & \multirow[t]{2}{*}{ GLY } & \multicolumn{3}{|c|}{ PAR } & TWA & \multicolumn{2}{|l|}{ GLU } & \multicolumn{2}{|l|}{ GLY } & \multicolumn{2}{|l|}{ PAR } & \multirow[t]{2}{*}{ TWA } \\
\hline PS & \multicolumn{12}{|c|}{ First count of germination test (FCGT, \% normal seedlings) } & & \\
\hline $\mathrm{Z}-83$ & 81.3 & $\mathrm{bA}^{*}$ & 56.0 & $\mathrm{~dB}$ & 33.0 & $\mathrm{dC}$ & $90.0^{(1)}$ & 64.7 & $\mathrm{cA}$ & 37.5 & $\mathrm{cB}$ & 10.5 & $\mathrm{dC}$ & $92.0^{(1)}$ \\
\hline $\mathrm{Z}-85$ & 86.0 & $\mathrm{bA}$ & 78.7 & $\mathrm{cB}$ & 61.3 & $\mathrm{cC}$ & & 67.3 & $\mathrm{cA}$ & 66.0 & $\mathrm{bA}$ & 32.0 & $\mathrm{cB}$ & \\
\hline $\mathrm{Z}-87$ & 84.0 & $\mathrm{bA}$ & 87.0 & bA & 78.5 & $\mathrm{bB}$ & & 86.0 & $\mathrm{bA}$ & 80.0 & $\mathrm{aB}$ & 67.0 & $\mathrm{bC}$ & \\
\hline \multirow[t]{2}{*}{$\mathrm{Z}-92$} & 90.0 & $\mathrm{aA}$ & 92.7 & $\mathrm{aA}$ & 86.0 & $\mathrm{aB}$ & & 91.0 & $\mathrm{aA}$ & 84.5 & $\mathrm{aB}$ & 88.0 & $\mathrm{aA}$ & \\
\hline & \multicolumn{14}{|c|}{ Germination test (GER, \% normal seedlings) } \\
\hline $\mathrm{Z}-83$ & 89.3 & $\mathrm{bA}$ & 73.3 & $\mathrm{cB}$ & 46.0 & $\mathrm{cC}$ & $91.3^{(1)}$ & 74.7 & $\mathrm{cA}$ & 47.0 & $\mathrm{cB}$ & 24.5 & $\mathrm{dC}$ & $92.0^{(1)}$ \\
\hline Z-85 & 89.3 & $\mathrm{bA}$ & 87.5 & bA & 84.0 & $\mathrm{bB}$ & & 81.3 & $\mathrm{bA}$ & 72.5 & $\mathrm{bB}$ & 52.0 & $\mathrm{cC}$ & \\
\hline $\mathrm{Z}-87$ & 86.5 & $\mathrm{bA}$ & 88.5 & bA & 85.0 & bA & & 93.3 & $\mathrm{aA}$ & 86.0 & $\mathrm{aB}$ & 72.5 & $\mathrm{bC}$ & \\
\hline \multirow{2}{*}{ Z-92 } & 94.0 & $\mathrm{aA}$ & 96.0 & $\mathrm{aA}$ & 89.5 & $\mathrm{aB}$ & & 93.0 & $\mathrm{aA}$ & 87.0 & $\mathrm{aB}$ & 91.5 & $\mathrm{aA}$ & \\
\hline & \multicolumn{14}{|c|}{ Seedling shoot length (SL, cm) } \\
\hline $\mathrm{Z}-83$ & 3.3 & $\mathrm{bA}$ & 2.4 & $\mathrm{cB}$ & 2.4 & $\mathrm{cB}$ & $3.7^{\mathrm{ns}}$ & 2.5 & $\mathrm{cA}$ & 2.0 & $\mathrm{bB}$ & 2.5 & $\mathrm{bA}$ & $3.5^{(1)}$ \\
\hline $\mathrm{Z}-85$ & 3.8 & $\mathrm{aA}$ & 3.2 & $\mathrm{aB}$ & 2.9 & $\mathrm{bB}$ & & 3.2 & $\mathrm{bA}$ & 2.6 & $\mathrm{aB}$ & 2.7 & $\mathrm{bB}$ & \\
\hline $\mathrm{Z}-87$ & 3.2 & $\mathrm{bB}$ & 2.8 & $\mathrm{bC}$ & 3.8 & $\mathrm{aA}$ & & 3.7 & $\mathrm{aA}$ & 3.0 & $\mathrm{aB}$ & 3.1 & $\mathrm{aB}$ & \\
\hline \multirow[t]{2}{*}{ Z-92 } & 3.2 & $\mathrm{bC}$ & 3.6 & $\mathrm{aB}$ & 4.1 & $\mathrm{aA}$ & & 3.0 & $\mathrm{bB}$ & 2.9 & $\mathrm{aB}$ & 3.6 & $\mathrm{aA}$ & \\
\hline & \multicolumn{14}{|c|}{ Seedling radicle length $(\mathrm{RL}, \mathrm{cm})$} \\
\hline $\mathrm{Z}-83$ & 6.0 & $\mathrm{aA}$ & 3.0 & $\mathrm{~dB}$ & 2.9 & $\mathrm{cB}$ & $6.2^{(1)}$ & 4.9 & $\mathrm{cA}$ & 2.0 & $\mathrm{bC}$ & 3.1 & $\mathrm{bB}$ & $5.8^{(1)}$ \\
\hline $\mathrm{Z}-85$ & 6.4 & $\mathrm{aA}$ & 5.1 & $\mathrm{cB}$ & 3.6 & $\mathrm{bC}$ & & 5.7 & $\mathrm{bA}$ & 2.4 & $\mathrm{bB}$ & 2.5 & $\mathrm{bB}$ & \\
\hline $\mathrm{Z}-87$ & 6.9 & $\mathrm{aA}$ & 6.2 & $\mathrm{bB}$ & 6.2 & $\mathrm{aB}$ & & 6.6 & $\mathrm{aA}$ & 4.8 & $\mathrm{aB}$ & 4.0 & $\mathrm{aC}$ & \\
\hline \multirow[t]{2}{*}{ Z-92 } & 6.5 & $\mathrm{aA}$ & 6.9 & $\mathrm{aA}$ & 5.9 & $\mathrm{aB}$ & & 6.2 & $\mathrm{aA}$ & 5.2 & $\mathrm{aB}$ & 4.5 & $\mathrm{aC}$ & \\
\hline & \multicolumn{14}{|c|}{ Seedling dry mass (SDM, mg) } \\
\hline $\mathrm{Z}-83$ & 31.4 & $\mathrm{aA}$ & 31.3 & $\mathrm{aA}$ & 30.3 & bA & $31.6^{\mathrm{ns}}$ & 26.6 & $\mathrm{bA}$ & 26.3 & $\mathrm{aA}$ & 27.4 & $\mathrm{bA}$ & $26.4^{\mathrm{ns}}$ \\
\hline Z-85 & 26.7 & $\mathrm{bB}$ & 28.4 & $\mathrm{aB}$ & 33.7 & $\mathrm{aA}$ & & 26.9 & $\mathrm{bA}$ & 27.0 & $\mathrm{aA}$ & 25.1 & $\mathrm{bA}$ & \\
\hline $\mathrm{Z}-87$ & 27.3 & $\mathrm{bB}$ & 32.6 & $\mathrm{aA}$ & 27.5 & $\mathrm{bB}$ & & 26.6 & $\mathrm{bA}$ & 25.2 & $\mathrm{aA}$ & 26.0 & $\mathrm{bA}$ & \\
\hline Z-92 & 31.8 & $\mathrm{aA}$ & 30.5 & $\mathrm{aA}$ & 29.7 & bA & & 30.8 & $\mathrm{aA}$ & 28.5 & $\mathrm{aA}$ & 31.4 & $\mathrm{aA}$ & \\
\hline
\end{tabular}

${ }^{1}$ Herbicides (HER), phenological stage (PS), glufosinate-ammonium (GLU), glyphosate (GLY), paraquat (PAR), treatment without application (TWA). ${ }^{*}$ Lowercase letters (PS within each HER) and uppercase (HER withim each PS) distinct differ[Scott-Knott $\left.(\mathrm{P} \leq 0.05)\right]$. Results of the contrast [Scheffé (P $\leq$ $0.05)]$ are favorable to treatment without application ${ }^{(1)}$, treatment with herbicides ${ }^{(2)}$ and not significant ${ }^{(\mathrm{ns})}$.

respectively). In two early stages (Z-83 and Z-85) the applications of glufosinate-ammonium originated seedlings of largest SL; in contrast, to the last two application stages (Z-87 and Z-92), paraquat applications have higher seedling. The Z-92 stage did not statistically differ from TWA in SL. As for SDM, the applications in the Z-83 and Z-92 stages are independent of the herbicide used; the treatment with paraquat applied to Z-85 resulted in heavier seedlings, as well as, glyphosate in the Z-87 stage. Seedling radicle length (RL) was also influenced by 
Table 5. Characters related to seedling morphology and physiological quality of wheat (BRS Parrudo and TBIO Sinuelo) emerged in soil with application of non-selective herbicide in the pre-harvest.

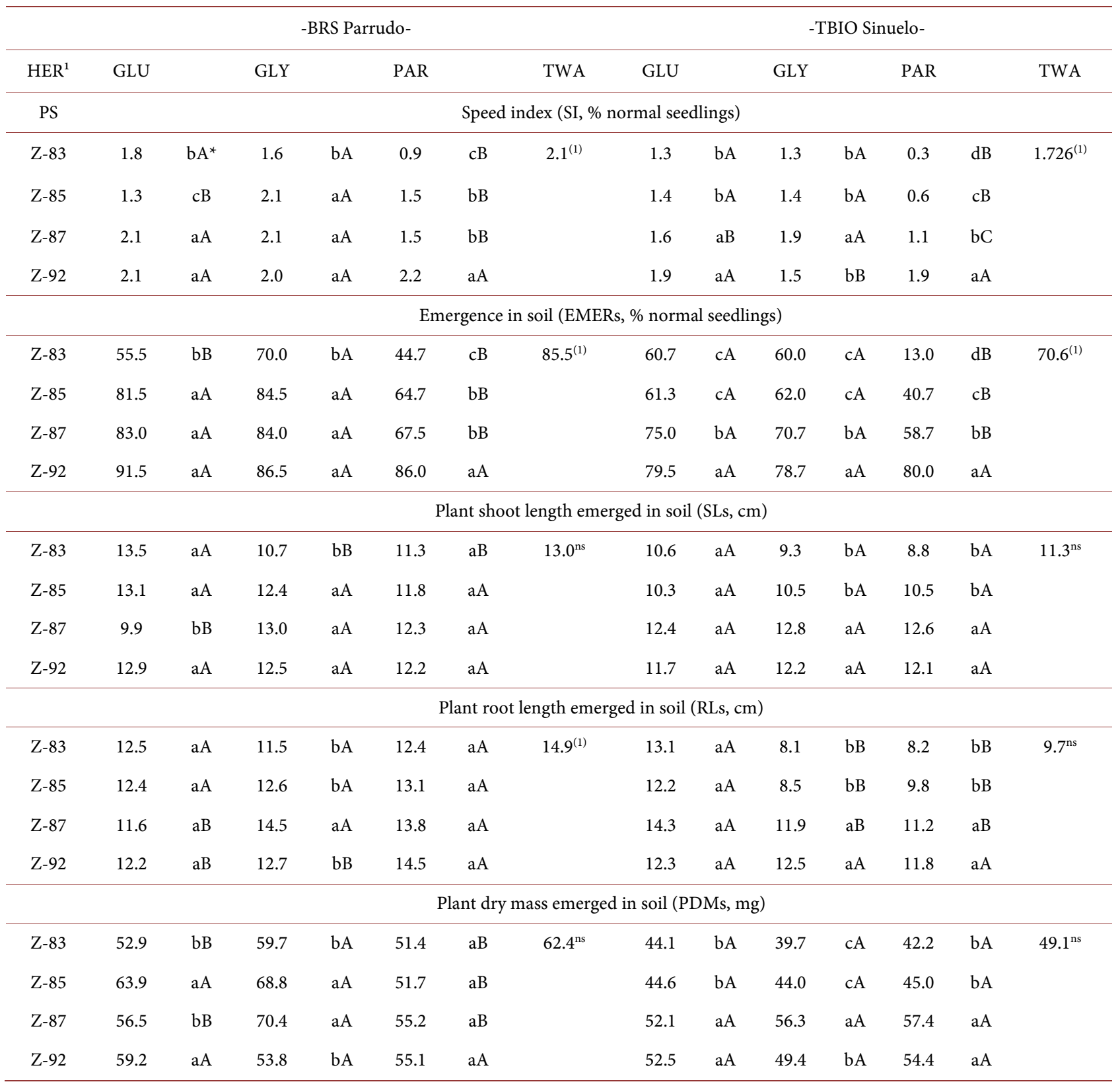

${ }^{1}$ Herbicides (HER), phenological stage (PS), glufosinate-ammonium (GLU), glyphosate (GLY), paraquat (PAR), treatment without application (TWA). ${ }^{*}$ Lowercase letters (PS within each HER) and uppercase (HER withim each PS) distinct differ [Scott-Knott $\left.(\mathrm{P} \leq 0.05)\right]$. Results of the contrast [Scheffé (P $\leq$ $0.05)]$ are favorable to treatment without application ${ }^{(1)}$, treatment with herbicides ${ }^{(2)}$ and not significant ${ }^{(\mathrm{ns})}$.

herbicides, differing statistically from TWA, except for the Z-87 and Z-92 stages. In general, the herbicide glufosinate-ammonium independently of the phenological stage of application originated seedlings with higher roots length.

The TWA presented 16\% higher germination speed index (SI) than herbicide treatments, differing statistically for the herbicide paraquat, as well as, the Z-83 stage (Table 5). The two initial stages presented the smallest indexes, except for the herbicide glyphosate. Emergence in soil (EMERs) was 14\% higher for TWA 
Table 6. Characters related to seed vigor in stress conditions of wheat (BRS Parrudo e TBIO Sinuelo) with application of non-selective herbicide in the pre-harvest.

\begin{tabular}{|c|c|c|c|c|c|c|c|c|c|c|c|c|c|c|}
\hline \multirow[b]{2}{*}{$\mathrm{HER}^{1}$} & \multicolumn{7}{|c|}{-BRS Parrudo- } & \multicolumn{7}{|c|}{-TBIO Sinuelo- } \\
\hline & \multicolumn{2}{|l|}{ GLU } & \multicolumn{2}{|l|}{ GLY } & \multicolumn{2}{|l|}{ PAR } & TWA & \multicolumn{2}{|l|}{ GLU } & \multicolumn{2}{|l|}{ GLY } & \multicolumn{2}{|l|}{ PAR } & \multirow[t]{2}{*}{ TWA } \\
\hline PS & \multicolumn{13}{|c|}{ Cold test (CT, \% normal seedlings) } & \\
\hline $\mathrm{Z}-83$ & 95.5 & $\mathrm{aA}^{*}$ & 86.7 & $\mathrm{bB}$ & 58.0 & $\mathrm{cC}$ & $93.5^{\mathrm{ns}}$ & 80.5 & $\mathrm{bA}$ & 48.0 & $\mathrm{cB}$ & 34.0 & $\mathrm{dC}$ & $96.0^{(1)}$ \\
\hline $\mathrm{Z}-85$ & 98.0 & $\mathrm{aA}$ & 93.5 & $\mathrm{aB}$ & 84.7 & $\mathrm{bC}$ & & 88.0 & $\mathrm{bA}$ & 72.0 & $\mathrm{bB}$ & 51.0 & $\mathrm{cC}$ & \\
\hline $\mathrm{Z}-87$ & 94.5 & $\mathrm{aA}$ & 93.0 & $\mathrm{aA}$ & 84.5 & $\mathrm{bB}$ & & 97.0 & $\mathrm{aA}$ & 91.5 & $\mathrm{aA}$ & 77.0 & $\mathrm{bB}$ & \\
\hline \multirow[t]{2}{*}{ Z-92 } & 96.5 & $\mathrm{aA}$ & 95.5 & $\mathrm{aA}$ & 97.5 & $\mathrm{aA}$ & & 94.5 & $\mathrm{aA}$ & 92.5 & $\mathrm{aA}$ & 94.0 & $\mathrm{aA}$ & \\
\hline & \multicolumn{14}{|c|}{ Accelerate aging test (AA, \% normal seedlings) } \\
\hline $\mathrm{Z}-83$ & 71.0 & $\mathrm{bA}$ & 73.3 & ba & 25.5 & $\mathrm{cB}$ & $78.0^{\mathrm{ns}}$ & 54.0 & $\mathrm{aA}$ & 35.3 & $\mathrm{cB}$ & 6.5 & $\mathrm{dC}$ & $72.5^{(1)}$ \\
\hline $\mathrm{Z}-85$ & 86.7 & $\mathrm{aA}$ & 86.0 & $\mathrm{aA}$ & 64.7 & $\mathrm{bB}$ & & 63.3 & $\mathrm{aA}$ & 51.5 & $\mathrm{bB}$ & 22.0 & $\mathrm{cC}$ & \\
\hline $\mathrm{Z}-87$ & 82.5 & $\mathrm{aA}$ & 84.7 & $\mathrm{aA}$ & 68.7 & $\mathrm{bB}$ & & 59.0 & $\mathrm{aA}$ & 67.3 & $\mathrm{aA}$ & 35.3 & $\mathrm{bB}$ & \\
\hline \multirow[t]{2}{*}{ Z-92 } & 79.3 & $\mathrm{aB}$ & 80.7 & $\mathrm{aB}$ & 88.7 & $\mathrm{aA}$ & & 62.7 & $\mathrm{aA}$ & 70.5 & $\mathrm{aA}$ & 66.0 & $\mathrm{aA}$ & \\
\hline & \multicolumn{14}{|c|}{ Electrical conductivitytest $\left(\mathrm{EC}, \mu \mathrm{S} \cdot \mathrm{cm}^{-1} \cdot \mathrm{g}^{-1}\right)$} \\
\hline $\mathrm{Z}-83$ & 12.0 & $\mathrm{bC}$ & 22.5 & $\mathrm{aA}$ & 14.7 & $\mathrm{bB}$ & $8.9^{(2)}$ & 13.8 & $\mathrm{aB}$ & 18.8 & $\mathrm{bA}$ & 15.2 & $\mathrm{bB}$ & $8.2^{(2)}$ \\
\hline Z-85 & 14.4 & $\mathrm{aB}$ & 18.8 & $\mathrm{bA}$ & 17.5 & $\mathrm{aA}$ & & 13.3 & $\mathrm{aC}$ & 21.0 & $\mathrm{aA}$ & 17.6 & $\mathrm{aB}$ & \\
\hline $\mathrm{Z}-87$ & 8.3 & $\mathrm{cB}$ & 10.2 & $\mathrm{cA}$ & 9.9 & $\mathrm{cA}$ & & 7.6 & $\mathrm{bA}$ & 8.7 & $\mathrm{cA}$ & 7.4 & $\mathrm{cA}$ & \\
\hline Z-92 & 9.8 & $c \mathrm{~A}$ & 10.5 & $\mathrm{cA}$ & 8.9 & $c \mathrm{~A}$ & & 8.0 & $\mathrm{bA}$ & 9.1 & $c A$ & 8.7 & $\mathrm{cA}$ & \\
\hline
\end{tabular}

${ }^{1}$ Herbicides (HER), phenological stage (PS), glufosinate-ammonium (GLU), glyphosate (GLY), paraquat (PAR), treatment without application (TWA). ${ }^{*}$ Lowercase letters (PS within each HER) and uppercase (HER withim each PS) distinct differ [Scott-Knott $(\mathrm{P} \leq 0.05)$ ]. Results of the contrast [Scheffé (P $\leq$ $0.05)]$ are favorable to treatment without application ${ }^{(1)}$, treatment with herbicides ${ }^{(2)}$ and not significant ${ }^{(\mathrm{ns})}$.

and did not differ statistically for the Z-83, Z-87 and Z-92 stages and also for the herbicides glufosinate-ammonium and glyphosate. However, the results of plant shoot lenght (SLs), dry mass (PDMs) and root lenght (RLs) emerged in soil, coincided with the characters measured in laboratory conditions, i.e., they did not differ statistically for the first two and there were 16\% (TWA) for RLs.

To the cold test (CT), TWA did not differ statistically from herbicide treatments, except for the Z-83 stage and the herbicide paraquat, for these conditions the TWA obtained increases of $16 \%$ and $15 \%$, respectively (Table 6). For the accelerated aging test (AA) there was a reduction for the herbicide paraquat (26\%) and the Z-83 stage (37\%). The electrical conductivity test (EC) showed $47 \%$ difference between TWA and herbicide treatments.

For the blotter test (BT) there was no statistical difference between the contrasts, but the herbicide paraquat reduced the incidence of fungi by $11 \%$ when compared to the TWA (Table 7). There were 53\% and 26\% incidence of Fusarium sp. (FUS) and Cladosporium cladosporioides (CLA), respectively. There was a $31 \%$ reduction in the incidence of Alternaria alternata (ALT) for TWA in relation to herbicide treatments. 
Table 7. Percentage of incidence of wheat pathogens (BRS Parrudo and TBIO Sinuelo) and estimates of the contrasts of treatments with non-selective herbicides applied in the wheat pre-harvest (Z-92 stage) versus the treatment without application.

\begin{tabular}{|c|c|c|c|c|c|c|c|c|}
\hline Herbicides & $\mathrm{GLU}^{1}$ & GLY & PAR & TWA & GLU & GLY & PAR & TWA \\
\hline Pathogens & \multicolumn{4}{|c|}{-BRS Parrudo- } & \multicolumn{4}{|c|}{-TBIO Sinuelo- } \\
\hline FUS & $53.9 \mathrm{aA}^{*}$ & $52.0 \mathrm{aA}$ & $54.4 \mathrm{aA}$ & $53.3 \mathrm{aA}$ & $53.9 \mathrm{aB}$ & $58.94 \mathrm{aA}$ & $63.2 \mathrm{cA}$ & $61.5 \mathrm{aA}$ \\
\hline ALT & $16.8 \mathrm{cA}$ & $19.4 \mathrm{cA}$ & $16.7 \mathrm{cA}$ & $13.4 \mathrm{cB}$ & $27.8 \mathrm{bA}$ & $21.8 \mathrm{bB}$ & $19.8 \mathrm{bB}$ & $18.1 \mathrm{bB}$ \\
\hline ASP & $0.0 \mathrm{dA}$ & $0.0 \mathrm{dA}$ & $0.0 \mathrm{dA}$ & $0.3 \mathrm{dA}$ & $0.0 \mathrm{dA}$ & $0.0 \mathrm{dA}$ & $0.0 \mathrm{cA}$ & $0.4 \mathrm{cA}$ \\
\hline CLA & $25.9 \mathrm{bA}$ & $24.3 \mathrm{bA}$ & $28.5 \mathrm{bA}$ & $28.5 \mathrm{bA}$ & $17.5 \mathrm{cA}$ & $16.3 \mathrm{cA}$ & $16.6 \mathrm{bA}$ & $19.4 \mathrm{bA}$ \\
\hline CUR & $2.8 \mathrm{dA}$ & $3.3 \mathrm{dA}$ & $1.5 \mathrm{dA}$ & $1.5 \mathrm{dA}$ & $2.5 \mathrm{dA}$ & $2.2 \mathrm{dA}$ & $0.8 \mathrm{cA}$ & $0.4 \mathrm{cA}$ \\
\hline BIP & $0.0 \mathrm{dA}$ & $0.3 \mathrm{dA}$ & $0.0 \mathrm{dA}$ & $0.0 \mathrm{dA}$ & $0.4 \mathrm{dA}$ & $0.8 \mathrm{dA}$ & $0.0 \mathrm{cA}$ & $0.0 \mathrm{cA}$ \\
\hline $\mathrm{PHO}$ & $2.1 \mathrm{dA}$ & $0.7 \mathrm{dA}$ & $2.7 \mathrm{dA}$ & $2.7 \mathrm{dA}$ & $0.0 \mathrm{dA}$ & $0.7 \mathrm{dA}$ & $0.9 \mathrm{cA}$ & $1.7 \mathrm{cA}$ \\
\hline S.V. & D.F. & & Meansquares & & & & eansquare & \\
\hline Treatments & 3 & & $1.93^{\mathrm{ns}}$ & & & & $0.18^{\text {ns }}$ & \\
\hline Pathogens & 6 & & $6375.9^{*}$ & & & & $892.7^{\star}$ & \\
\hline Trat $\mathrm{x}$ Path. & 18 & & $8.42^{\mathrm{ns}}$ & & & & $2.98^{\text {ns }}$ & \\
\hline Error & 84 & & 7.25 & & & & 8.49 & \\
\hline Average & & & 18.58 & & & & 14.51 & \\
\hline \multirow[t]{2}{*}{$\mathrm{CV}(\%)$} & & & 14.50 & & & & 20.08 & \\
\hline & \multicolumn{8}{|c|}{-Contrasts- } \\
\hline Treatments & & & $0.22^{\mathrm{ns}}$ & & & & $-0.06^{\mathrm{ns}}$ & \\
\hline glufosinate & & & $0.23^{\mathrm{ns}}$ & & & & $-0.08^{\mathrm{ns}}$ & \\
\hline glyphosate & & & $-0.07^{\mathrm{ns}}$ & & & & $-0.10^{\mathrm{ns}}$ & \\
\hline paraquat & & & $0.51^{\mathrm{ns}}$ & & & & $0.08^{\mathrm{ns}}$ & \\
\hline
\end{tabular}

${ }^{1}$ Herbicides: glufosinate-ammonium (GLU), glyphosate (GLY), paraquat (PAR), treatment without application (TWA). Source of variation (S.V.), degree of freedom (D.F.), coefficient of variation (CV\%). Pathogens: Fusarium sp. (FUS), Alternariaalternata (ALT), Aspergillus sp. (ASP), Cladosporiumcladosporioides (CLA), Curvularia luneta (CUR), Bipolarissorokiniana (BIP), Phoma sp. (PHO). * Lowercase letters (pathogens within each herbicide) and uppercase (herbicides withim each pathogen) distinct differ $[$ Scott-Knott $(\mathrm{P} \leq 0.05)]$ and results of contrast [Scheffé $(\mathrm{P} \leq 0.05)]$ not significant ${ }^{(\mathrm{ns})}$.

\subsection{TBIO Sinuelo}

The FCGT was $42 \%$ higher for the TWA than the others (Table 4). The phenological stage of application that provided the lowest reduction was Z-92 for all the herbicides tested and there is reduction of this character with the anticipation for earlier stages. Glufosinate-ammonium presented a $15 \%$ and $56 \%$ increase in relation to glyphosate and paraquat, respectively. The GER followed this same response, with $26 \%$ reduction in herbicide treatments, reduction in the first application stages, and an increase (with no statistical difference in relation to TWA) at the Z-92 stage. The SL $(3.5 \mathrm{~cm})$ and RL $(5.8 \mathrm{~cm})$ showed a 23 and $34 \%$ increase in relation to herbicide treatments (2.9 and 4.3 , respectively). For SDM, however, did not differ statistically; however, in absolute numbers to the Z-92 $(30.2 \mathrm{mg})$ applications, weighed seedlings were obtained in relation to the control (26.4 mg). 
In absolute numbers, there was emphasis on the Z-92 stage (79\%) for the EMERs in relation to TWA (70\%), however, no differ statistically; in none of the treatments tested was reached the minimum requirement for use as seeds. For the SLs, RLs and PDMs, there was no statistical difference, except for the herbicide glufosinate-ammonium, as well as, Z-87 and Z-92, which had 33\%, 28\% and $25 \%$ higher plant roots than the control.

Germination in CT and AA was higher in TWA treatment (25\% and $46 \%$, respectively) compared to herbicides treatment (Table 6). Applications on Z-92 stage (93\% and 66\%) does not interfere in these characters, when compared to their respective plots without application (96\% and $72 \%$ ). The deterioration in seeds imposed by application of herbicides was evidenced by present EC twice against TWA, except for the Z-87 stage and Z-92 which did not differ statistically. For BT there were no statistical differences between the contrasts; $(58 \%)$ and ALT (23\%), which presented a double incidence (53\%) for treatment with glufosinate-ammonium compared to TWA (Table 7).

\section{Discussion}

The results from the FCGT and GER tests showed the same relation in their means, with a reduction of the physiological potential with applications previous to the Z-87 stage, as well as, the use of glyphosate and paraquat herbicides. These two herbicides affect wheat germination, with a more pronounced effect for the second that is $7 \%$ more harmful than glyphosate [4]. The glufosinate-ammonium when applied in pre-harvest with 26,33, 40 days after flowering does not affect the germination in relation to control, without application [12]. On the other hand, doses from $1 \mathrm{~kg} \cdot \mathrm{ha}^{-1}$ of glyphosate applied to winter wheat at the Z-87 stage reduce initial vigor, germination and increase the occurrence of abnormal seedlings [26].

As for the application stage, [4] obtained an increase in germination in early applications, as in stage 11.2 [27]. This phenological stage comprises the interval between stage Z-83 and Z-87, in this way, opposing the results of the present study. The authors report that application at a later phenological stage was compromised by adverse climatic conditions during the harvest period. Sustaining this narrative, [13] also claim that there is no reduction in germination, since the seeds are in physiological maturity, $34 \%-40 \%$ seed moisture and with plant tissues of yellowing. The application with moisture contents of the seed, such as $49 \%-53 \%$, reduces germination by up to $70 \%$ [28]. Comparing with our results, we can infer that the pre-harvest application of the herbicide glufosinate-ammonium does not affect the physiological quality expressed by the vigor and germination of seeds in the wheat crop. However, the most efficient application stage does not correspond to a satisfactory harvest anticipation period, and its technical and economic viability is questionable.

The SL and RL are decreased by the application of herbicides, regardless of the phenological stage of application, and there is a tendency for the maintenance of 
growth expression when using glufosinate-ammonium applied in Z-92. There is a negative influence among herbicides compared to TWA, because seedlings with shoot and smaller rootlets are obtained [4]. For glyphosate, radicle damage is much higher in relation to shoot, with an average reduction of $2.5 \mathrm{~cm}$ in relation to treatments without the application of herbicides [26]. In relation to the application of this herbicide in post-emergence, the shikimic acid content rises in all the parts of plant, accumulating in the root, stem and leaves. This concentration continues to increase in the roots until seven days after application, while in the other parts of the plant, it decreases [5]. This accumulation in the roots may be related with the subsequent rise of seedlings with minor leaf and root primitives. The SDM was not affected by the use of herbicides, regardless of the application stage. From the Z-83 stage, [4] report that there is reduction in the mass accumulation of plants coming from desiccation. The dry mass is $10 \%$ smaller with use of $2 \mathrm{~kg} \cdot \mathrm{ha}^{-1}$ of glyphosate, with further reduction (17\%) in the rootlets, specifically [26]. In the present study, the methodology used in the measurement of the dry mass may have contributed to the fact that no statistical difference was found between the treatments. Firstly, only normal seedlings were chosen, even though the rate of occurrence of the abnormal ones was much higher. Later, it would be more plausible to remove the seed for measurement, since it represents a large part of the mass of seedlings with only four days.

There were reductions in the IS and EMERs inherent in the use of herbicides; the results followed the trend of tests expressing initial vigor and final germination (FCGT and GER). Smaller reductions were observed with use of glufosinate-ammonium and applications on Z-92 stage. In sand, seed emergence from plants desiccated with gliphosate at the Z-87 stage, when allocated up to $4 \mathrm{~cm}$ deep, did not differ statistically from the non-desiccated control [26]. With application to the milky grain stage (Z-79), [29] report that there is a $42 \%$ reduction in initial establishment rate and in the final plant stand due to low germination. For the conditions of the present study it is important to emphasize that the cultivar TBIO Sinuelo presented EMERs very reduced, below the $80 \%$ required by the legislation [30]. However, it was observed that this fact was not due to the pre-harvest application, because the TWA also presented germination below the required standard.

For growth characters measured in soil conditions (SLs, RLs and PDMs) there were no interferences from the use of herbicides. However, when comparing cultivars, BRS Parrudo obtained larger SLs in the TWA treatment, and TBIO Sinuelo in the treatments with glufosinate-ammonium applied in the Z-87 and Z-92 stages. The size of the coleoptile can be affected, generating smaller seedlings, however, this is an inconsistent evaluation to express the vigor. Plants with more than one week are necessary for shoot and root evaluation [29]. The harmful effects of herbicide seed germination are inhibited under sand substrate conditions and do not differ statistically from the control treatment (without application) on shoot length and radicle, as well as, on the mass accumulation of 
both structures [26]. Once again, the methodology used in our study may have contributed to the conflicting results with the literature. Plants with 20 days were used, i.e. they weren't being nourished by the reserves of the seed, and variability may have arisen by conditions imposed by the environment.

The tests that express the physiological potential under adverse stress conditions (CT and AA) differed in terms of plant cultivars. For BRS Sinuelo, both tests did not differ from TWA and TBIO Sinuelo treatment with herbicides have damaged the germination. Using the cultivar Quartzo, [4] report the toxic effect caused by their herbicides under germination by the same two tests, corroborating with the results of the present study for the cultivar TBIO Sinuelo. In order, to separate wheat lots and cultivars, the cold test is not suitable for evaluating the vigor of wheat seeds [31]. The authors report that the test is related to germination only when the seeds with visible germination start are used. Thus, the use of only those seeds that initiate the protrusion of the primary root at seven days at $5^{\circ} \mathrm{C}$ is indicated. This may have contributed because different results were obtained at the cultivar level. Already for the vigor expressed by AA, there is a close relationship with germination. The deterioration rate of low quality seeds is stimulated by exposure to high temperature and relative humidity [32]. Therefore, for the BRS Parrudo it can be affirmed that the application of herbicides in the pre-harvest does not change the quality expressed by this character.

Higher seed deterioration was evidenced by high EC measured in herbicide treatments, regardless of the active ingredient in the first two application stages (Z-83 and Z-85). These results corroborate [4] with application of the herbicides glyphosate and paraquat in the pre-harvest, which directly interferes with the vigor and germination of wheat seeds, presenting absolute values up to $34 \mu \mathrm{S}$ $\mathrm{cm}^{-1} \cdot \mathrm{g}^{-1}$. The authors cite the most deleterious effect of glyphosate herbicide when applied in advance of paraquat when applied closest to harvest. The time of reestablishment of the integrity of the seed membranes embedded in the solution and consequent release of solutes into the external environment is measured by the EC test [33]. In this way, we can infer that the phenological stage in which pre-harvest herbicides are applied is decisive for obtaining vigorous (less deteriorated) seeds.

The fungal incidence measured by BT was not affected by herbicide treatments, with presence of pathogens in any of the conduction conditions of the experiment. The pathogen F. graminearum (teleomorph Giberellazeae), causes giberela in cereals, is widely disseminated in the agricultural field areas, and was present in practically all the samples evaluated in our experiment. High levels of rainfall during the harvest period contribute to the infection of seeds [34]. Infected seed dispersal is characterized as the main source of inoculum, briefly rendering the cultivation unfeasible [20]. In our study, comparison was made only between herbicide treatments and TWA. The application phenological stages were disregarded because the storage period of seeds harvested first, could contribute to a higher incidence of pathogens. Therefore, seeds from the Z-92 
application were used, which were harvested the same week as the TWA. Rainfall that occurred in this period was the same for all treatments. That is, there is no influence between herbicides and the incidence of pathogens.

The quality of seeds from plants where pre-harvest herbicide applications were strongly influenced by the phenological stage and the proximity of phenological maturation at the time of application than by the dose of herbicide or cultivar [29]. According to the most of our results, this practice is detrimental to vigor and seed germination. If, there are agronomic needs to anticipate the wheat harvest, one should choose glufosinate-ammonium from the Z-87 stage. In this way, there will be little reduction in the physiological quality of seeds.

\section{Conclusions}

The application of non-selective herbicides on wheat pre-harvest impairs the physical and physiological quality and promotes faster deterioration. Vigor is reduced under stress conditions by cold test and accelerated aging. There is no influence on seed sanity.

In general, applications in phenological stages prior to Z-87 stage, especially with the herbicide paraquat should be avoided.

\section{Acknowledgements}

The authors would like to thank the Higher-Level Personnel Improvement Coordination (Capes-Brazil) for the scholarship provided, The National Council for Scientific and Technological Development (CNPq).

\section{References}

[1] Pereira, T., Coelho, C.M.M., Souza, C.A., Mantovani, A. and Mathias, V. (2015) Dessecação Química Para Antecipação de Colheita Em Cultivares de Soja. Semina: Ciências Agrárias, 36, 2383. https://doi.org/10.5433/1679-0359.2015v36n4p2383

[2] Pires, J.L.F., Vargas, L. and Cunha, G.R. (2011) Trigo No Brasil - Bases Para a Produção Competitiva E Sustentável. Passo Fundo.

[3] Carneiro, L.M.T.A., Biagi, J.D., De Freitas, J.G., Carneiro, M.C. and Felício, J.C. (2005) Diferentes Épocas de Colheita, Secagem E Armazenamento Na Qualidade de Grãos de Trigo Comum E Duro. Bragantia, Instituto Agronômico, 127-137. https://doi.org/10.1590/S0006-87052005000100014

[4] Bellé, C., Kulczynski, S.M., Basso, C.J., Edu Kaspary, T., Lamego, F.P. and Pinto, M.A.B. (2014) Yield and Quality of Wheat Seeds as a Function of Desiccation Stages and Herbicides. Journal of Seed Science, ABRATES - Associação Brasileira de Tecnologia de Sementes, 36, 63-70. https://doi.org/10.1590/S2317-15372014000100008

[5] Bresnahan, G.A., Manthey, F.A., Howatt, K.A. and Chakraborty, M. (2003) Glyphosate Applied Preharvest Induces Shikimic Acid Accumulation in Hard Red Spring Wheat (Triticum aestivum). Journal of Agricultural and Food Chemistry, 51, 4004-4007. https://doi.org/10.1021/jf0301753

[6] Brasil. Ministério da Agricultura Pecuária e Abastecimento (2017) Sistema de Agrotóxicos Fitossanitários.

http://agrofit.agricultura.gov.br/agrofit_cons/principal_agrofit_cons 
[7] Griffin, J.L., Boudreaux, J.M. and Miller, D.K. (2010) Herbicides As Harvest Aids. Weed Science, 58, 355-358. https://doi.org/10.1614/WS-09-108.1

[8] Jablonkai, I. (2011) Molecular Mechanism of Action of Herbicides. In: Hasaneen, M.N., Ed., Herbicides - Mechanisms and Mode of Action, InTech, 3-24. https://doi.org/10.5772/31251

[9] Sherwani, S.I., Arif, I.A. and Khan, H.A. (2015) Modes of Action of Different Classes of Herbicides. In: Price, A., Kelton, J. and Sarunaite, L., Eds., Herbicides, Physiology of Action, and Safety, InTech, 165-186. https://doi.org/10.5772/61779

[10] Cessna, A.J., Darwent, A.L., Kirkland, K.A., Townley-Smith, L., Harker, K.N. and Lefkovitch, L. (1994) Residues of Glyphosate and Its Methabolite AMPA in Wheat Seed and Foliage Following Pre-Harves Application. Canadian Journal of Plant Science, 74, 653-661. https://doi.org/10.4141/cjps94-117

[11] Hanft, J.M. and Wych, R.D. (1982) Visual Indicators of Physiological Maturity of Hard Red Spring Wheat. Crop Science, 22, 584. https://doi.org/10.2135/cropsci1982.0011183X002200030036x

[12] Santos, P.R.R. and Vicente, D. (2009) Momento Fisiológico Das Plantas de Trigo Para a Dessecação E Seus Efeitos No Rendimento de Grãos. Cultivando o saber, 2, 52-62. https://www.fag.edu.br/upload/revista/cultivando_o_saber/591a1fee7d5fb.pdf

[13] Mellado, Z.M. and Pedreros, L.A. (2005) Efecto de Herbicidas Aplicados Durante La Madurez Del Grano de Trigo En El Rendimiento Y Calidad Del Grano. Agricultura Técnica, 65, 312-318. https://doi.org/10.4067/S0365-28072005000300009

[14] C. Q. F. S. Comissão de Química e Fertilidade do Solo (2016) Manual de Calagem E Adubação Para Os Estados Do Rio Grande Do Sul E Santa Catarina. 11th Edition, Porto Alegre.

[15] Embrapa. Empresa Brasileira de Pesquisa Agropecuária (2017) Informações Técnicas Para Trigo E Triticale.

http://ainfo.cnptia.embrapa.br/digital/bitstream/item/155787/1/Informacoes-Tecnic as-para-Trigo-e-Triticale-Safra-2017-OL.pdf

[16] Zadoks, J.C., Chang, T.T. and Konzak, C.F. (1974) A Decimal Code for the Growth Stages of Cereals. Weed Research, 14, 415-421.

https://doi.org/10.1111/j.1365-3180.1974.tb01084.x

[17] Brasil Ministério da Agricultura Pecuária e Abastecimento (2009) Regras Para Análise de Sementes. MAPA/ACS.

http://www.agricultura.gov.br/assuntos/insumos-agropecuarios/arquivos-publicaco es-insumos/2946_regras_analise_sementes.pdf/@@download/file/2946_regras_ana lise_sementes.pdf

[18] Nakagawa, J.T. (1999) Testes de Vigor Baseados No Desempenho Das Plântulas. In: Kryzanoski, F.C., Vieira, R.D. and Franca Neto, J.B., Eds., Vigor de Sementes. Conceitos E Testes, 164.

[19] Rampim, L., Rodrigues-Costa, A.C.P., Nacke, H., Klein, J. and Guimarães, V.F. (2012) Qualidade Fisiológica de Sementes de Três Cultivares de Trigo Submetidas À Inoculação E Diferentes Tratamentos. Revista Brasileira de Sementes, 34, 678-685. https://doi.org/10.1590/S0101-31222012000400020

[20] Garcia Júnior, D., Vechiato, M.H. and Menten, J.O.M. (2008) Efeito de Fungicidas No Controle de Fusarium Graminearum, Germinação, Emergência E Altura de Plântulas Em Sementes de Trigo. Summa Phytopathologica, 34, 280-283. https://doi.org/10.1590/S0100-54052008000300018

[21] Nogueira, J.L., Silva, B.A. da, Carvalho, T.C. de and Panobianco, M. (2013) Teste de 
Condutividade Elétrica Para Avaliação Do Potencial Fisiológico de Sementes de Aveia Preta. Revista Ceres, 60, 896-901. https://doi.org/10.1590/S0034-737X2013000600019

[22] Kobayasti, L. and Pires, A.P. (2011) Levantamento de Fungos Em Sementes de Trigo. Pesquisa Agropecuária Tropical, 41, 572-578.

https://doi.org/10.5216/pat.v41i4.12388

[23] Henning, A.A. (2005) Patologia E Tratamento de Sementes: Noções Gerais. Londrina: Embrapa Soja.

http://ainfo.cnptia.embrapa.br/digital/bitstream/CNPSO-2009-09/26692/1/docume nto264.pdf

[24] Cruz, C.D. (2013) GENES-A Software Package for Analysis in Experimental Statistics and Quantitative Genetics. Acta Scientiarum. Agronomy, 35, 271-276. https://doi.org/10.4025/actasciagron.v35i3.21251

[25] Ferreira, D.F. (2011) Sisvar: A Computer Statistical Analysis System. Ciência e Agrotecnologia, 35, 1039-1042. https://doi.org/10.1590/S1413-70542011000600001

[26] Jaskulski, D. and Jaskulska, I. (2014) The Effect of Pre-Harvest Glyphosate Application on Grain Quality and Volunteer Winter Wheat. Romanian Agricultural Research, 31, 283-289.

https://www.researchgate.net/publication/286226726_The_effect_of_pre-harvest_gl yphosate_application_on_grain_quality_and_volunteer_winter_wheat

[27] Large, E.C. (1954) Growth Stages in Cereals. Illustration Od the Feeks Scale. Plant Pathology, 3, 128-129. https://doi.org/10.1111/j.1365-3059.1954.tb00716.x

[28] Calviño, P.A., Studdert, G.A., Abbate, P.E., Andrade, F.H. and Redolatti, M. (2002) Use of Non-Selective Herbicides for Wheat Physiological and Harvest Maturity Acceleration. Field Crops Research, 77, 191-199.

https://doi.org/10.1016/S0378-4290(02)00072-2

[29] Yenish, J.P. and Young, F.L. (2000) Effect of Preharvest Glyphosate Application on Seed and Seedling Quality of Spring Wheat (Triticum aestivum). Weed Technology, 14, 212-217. https://doi.org/10.1614/0890-037X(2000)014[0212:EOPGAO]2.0.CO;2

[30] Brasil Ministério da Agricultura Pecuária e Abastecimento (2013) Instrução Normativa $\mathrm{n}^{\circ} 45$, de 17 de Setembro de 2013. Brasília, DF.

http://www.agricultura.gov.br/assuntos/insumos-agropecuarios/insumos-agricolas/ se-

mentes-e-mudas/publicacoes-sementes-e-mudas/INN45de17desetembrode2013.pdf

[31] Fanan, S., Medina, P.F., Lima, T.C. and Marcos Filho, J. (2006) Avaliação Do Vigor de Sementes de Trigo Pelos Tetes de Envelhecimento Acalerado E de Frio. Revista Brasileira de Sementes, 28, 152-158. https://doi.org/10.1590/S0101-31222006000200021

[32] Ohlson, O. de C., Krzyzanowski, F.C., Caieiro, J.T. and Panobianco, M. (2010) Teste de Envelhecimento Acelerado Em Sementes de Trigo. Revista Brasileira de Sementes, 32, 118-124. https://doi.org/10.1590/S0101-31222010000400013

[33] Mertz, L.M., Segalin, S.R., Huth, C. and Rosa, T.D.A. (2012) Condutividade Elétrica Individual Para a Avaliação Do Potencial Fisiológico de Sementes de Trigo. Informativo ABRATES, 22, 35-39. http://www.abrates.org.br/downloads/file/54

[34] Brzezinski, C.R., Zucareli, C., Henning, F.A., Abati, J., Prando, A.M. and Henning, A.A. (2014) Nitrogênio E Inoculação Com Azospirillum Na Qualidade Fisiológica E Sanitária de Sementes de Trigo. Revista de Ciências Agrarias-Amazon Journal of Agricultural and Environmental Sciences, 57, 257-265.

https://doi.org/10.4322/rca.ao1391 